\title{
Corela
}

Cognition, représentation, langage

15-1 | 2017

Vol. $15, \mathrm{n}^{\circ} 1$

\section{Album ou texte? Résultats comparés de deux modalités d'exposition à une même histoire lors d'une séance de langage en grande section de maternelle}

Pierre PÉROZ

\section{OpenEdition}

\section{Journals}

Édition électronique

URL : https://journals.openedition.org/corela/4905

DOI : 10.4000 /corela.4905

ISSN : 1638-573X

Éditeur

Cercle linguistique du Centre et de l'Ouest - CerLICO

Référence électronique

Pierre PÉROZ, «Album ou texte? Résultats comparés de deux modalités d'exposition à une même histoire lors d'une séance de langage en grande section de maternelle », Corela [En ligne], 15-1 | 2017, mis en ligne le 26 juin 2017, consulté le 21 septembre 2021. URL : http://journals.openedition.org/ corela/4905 ; DOI : https://doi.org/10.4000/corela.4905

Ce document a été généré automatiquement le 21 septembre 2021.

\section{(c) (i) (3)(2)}

Corela - cognition, représentation, langage est mis à disposition selon les termes de la licence Creative Commons Attribution - Pas d'Utilisation Commerciale - Partage dans les Mêmes Conditions 4.0 International. 


\title{
Album ou texte? Résultats comparés de deux modalités d'exposition à une même histoire lors d'une séance de langage en grande section de maternelle
}

\author{
Pierre PÉROZ
}

1 Les enseignants privilégient les textes de fiction et en particulier les albums comme support pour les séances de langage à l'école maternelle². Dans les années 90, Grossmann montrait que des recueils de contes traditionnels - édités chez Milan - ou écrits par des auteurs comme Lily Boulay ${ }^{2}$ se trouvaient encore dans le « répertoire » d'une partie des enseignants de maternelle. Mais cette partie lui semblait déjà appartenir à un courant qu'il qualifiait de « traditionnaliste » par opposition aux « modernistes », plus nombreux, qui leur préféraient les albums. Une nouvelle enquête montrerait sans aucun doute que les enseignants qui lisent ou racontent des histoires sans présenter en même temps un album à leurs élèves sont très peu nombreux ${ }^{3}$.

2 Les raisons de cet engouement sont assez évidentes. La variété, les qualités littéraires et esthétiques des albums assurent aux enseignants un choix d'histoires adaptées toujours à même de capter l'attention de leurs élèves, au moins dans un premier temps. Il est aussi communément admis que les illustrations facilitent la mémorisation et la compréhension des histoires lues aux élèves ${ }^{4}$. Enfin la rencontre avec les multiples formes de relations texte-images présentes dans les albums (Van Der Linden, 2006) devraient permettre de développer des compétences de lecture-complexe. Ils constituent ainsi une introduction à la lecture littéraire (Tauveron, 2002).

3 Pour l'apprentissage du langage oral, la question n'est donc pas l'album mais quel album ? Pour preuve, le nombre d'articles ou de publications qui proposent des analyses critiques de la production éditoriale. Il s'agit alors de prévenir les difficultés propres à telle ou telle configuration iconico-textuelle - c'était déjà le choix de Lentin (1983) -, c'est plus 
récemment celui des auteurs du groupe PROG (2006: 262-301). Ces études sont aussi souvent l'occasion de fournir aux enseignants une sélection de titres dans la production courante comme avait pu le faire Stoecklé (1999) dans L'album à l'école et au collège ou comme vient de le faire Canut (2012) dans Des albums pour apprendre à parler. Les choisir, les utiliser en maternelle.

Pour les enseignants qui pourront facilement trouver des titres correspondant à leurs besoins dans ces différents ouvrages, le présupposé est qu'à texte équivalent l'album apporte en sus un bénéfice pédagogique : l'intérêt des élèves pour les illustrations qui accompagnent le texte.

5 Mais est-on bien certain de cela? Quels seraient les effets sur les productions orales des élèves si l'on choisissait comme support un texte non illustré?

6 Les résultats de plusieurs recherches dans le domaine de l'acquisition viennent indirectement étayer notre questionnement. François (1977 : 181-189) montrait déjà que la présence des images sous les yeux des enfants de 4 à 5 ans débouche sur la réduction des prédicats verbaux dans des conduites narratives, des rappels de récit ou des récits libres. La rentabilité, sur le plan verbal, de la fonction d'ancrage mémoriel souvent attribuée aux illustrations mérite aussi d'être interrogée. Dans une étude parue en 2009, Veneziano montre qu'après l'écoute d'une histoire accompagnée d'images et racontée par un adulte, la procédure d'étayage la plus efficace pour un deuxième rappel de récit de la part des enfants est de leur raconter l'histoire une seconde fois tandis que leur montrer les images une deuxième fois ou leur poser des questions sur les points-clés de l'histoire débouchent sur des résultats inférieurs à la première procédure. De leur côté, Soler et Sole (2010 : 45) établissent que dans des tâches narratives, la longueur des récits d'enfants de 6 à 10 ans n'évolue pas lorsqu'ils travaillent sur des supports imagés alors que Colletta (2004:245) montre que sur la même période les récits d'événements évoluent tant pour la complexité du récit que pour sa durée.

7 Du côté des didacticiens, on s'est surtout intéressé aux difficultés de compréhension que présentent bien souvent les albums apparemment faciles (Boiron et Rebière, 2009). L'équipe PROG, sous la direction de Brigaudiot (2000), proposait déjà des procédures de dissociation des canaux textuel et iconique tout en préconisant le choix de textes «autonomes» qui n'ont donc pas besoin d'illustrations pour être compris, selon la définition de Grossmann (1996). Dans un article de 1993, Brigaudiot évoquait mais sans en tirer d'enseignements particuliers les très bons résultats obtenus par une enseignante qui s'était bornée à lire une histoire à ses élèves de GS à partir d'un texte non illustré, justement. Enfin, tout récemment, un article de Boiron (2014) montre que dans des conditions similaires d'exposition au récit - excepté le support -, une centaine d'élèves de différents niveaux de l'école maternelle mémorisent et comprennent mieux une histoire quand elle leur est racontée que lorsqu'on la leur a présentée lors d'une lecture d'album. Le rapprochement de ces observations faites à des périodes différentes et selon des points de vue différents montre que le choix des supports en langage mérite d'être interrogé.

8 La présence des illustrations lors de la lecture de l'album ou leur absence lors de la lecture d'un texte non illustré confrontent les élèves à la construction d'univers de référence différents. Les uns partagent le souvenir des illustrations qui accompagnaient la narration tandis que les autres ont n'ont eu accès qu'à la narration verbale. Cela induitil des différences identifiables dans les interventions orales des élèves? et sur quels plans? Pour répondre à cette question, nous proposons de comparer les résultats de deux séances de langage, conduites en grande section de maternelle ${ }^{5}$ (GS) dans des conditions 
similaires, au support près : un album dans un cas et un texte non illustré dans l'autre, en l'occurrence, le même que celui de l'album.

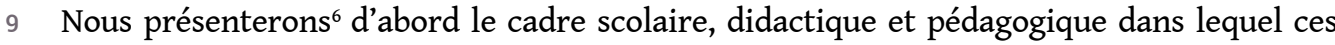
deux séances ont été menées. Puis, nous verrons quels en ont été les résultats sur les plans langagier et linguistique. La structure linéaire de l'exposé nous amène à présenter séparément nos observations, mais ce sont les convergences que nous pouvons noter, les rapprochements que nous pourrons faire qui nous permettront de proposer une réponse à la question posée.

\section{Contexte scolaire, didactique et pédagogique}

Les deux séances ont eu lieu en février-mars 2012 dans une école de campagne dans le nord-mosellan ${ }^{7}$ en grande section. L'enseignante stagiaire ${ }^{8}$ qui les a réalisées a conduit la première (désormais séance $\mathrm{A}$ ) avec un groupe de douze élèves en s'appuyant sur une lecture-présentation d'album : lecture à voix haute accompagnée de la présentation des doubles-pages illustrées, à partir de l'album L'enfant-fleur de V. Wagner (2007) aux éditions Hatier. Deux jours plus tard, elle a mené la seconde (désormais séance B) avec un groupe d'élèves équivalent, sur la base d'une lecture du texte de cet album qu'elle avait préalablement recopié sur une feuille de papier. Pour éviter les différents biais que cette organisation pouvait générer, les deux groupes ont été constitués par l'enseignante titulaire de la classe de manière équilibrée tant pour le nombre que pour le niveau des élèves ${ }^{9}$ et les élèves du deuxième groupe n'ont pas assisté à la séance réservée à leurs camarades du premier groupe. Le déroulement des deux séances a suivi le même principe : lecture-présentation de l'album ou lecture du texte aux élèves puis restitution de ce dont ils se rappelaient sur la base d'un questionnement ouvert, adressé à l'ensemble du groupe. Pour que les conditions d'exposition à l'histoire soient bien les mêmes, la lecture-présentation d'album n'a eu lieu qu'une fois, de même que la lecture du texte seul; après cela les illustrations n'ont pas été présentées de nouveau de même que le texte n'a pas été relu pendant le moment de langage proprement dit des séances $\mathrm{A}$ et $\mathrm{B}$.

11 Sur le plan pédagogique, la conduite des échanges a été définie de manière identique pour les deux séances. En l'occurrence, l'enseignante devait adopter une posture en retrait, écouter ses élèves, éviter les relances individuelles et suivre un schéma de questionnement simple faisant se succéder des questions ouvertes qui n'avaient pas à être déclinées en questions fermées, pour favoriser l'écoute entre élèves et éviter les travers d'une conduite directive des échanges. Ces questions qui visaient principalement à favoriser la prise de parole étaient a priori les suivantes : " De quoi vous rappelez-vous?", «S'agit-il d'une histoire vraie? », « Qui sont les personnages de l'histoire? » et « Qu'estce qui a changé entre le début et la fin de l'histoire?». Elles pouvaient être répétées et données dans un ordre différent en fonction de l'évolution des échanges. L'objectif de restitution de l'histoire était présenté aux élèves dès le début de la séance, il était donc pour eux le même dans les deux cas.

12 Sur le plan didactique, le choix du support s'est porté sur L'enfant-fleur, un album de facture classique avec un texte autonome mais qui est accompagné d'images figuratives dont le rôle est clairement illustratif. Texte et images sont présentés de manière régulière. Chaque double-page présente une illustration pleine page à gauche ou à droite avec un léger débord sur l'autre page qui porte le paragraphe de texte sur fond blanc, avec en accompagnement de certains paragraphes, la reprise iconique d'un objet ou d'un 
élément important de l'épisode : calebasse, tam-tam ou danseurs. L'histoire ${ }^{10}$, présentée comme un conte étiologique, explique l'arrivée de la pluie dans un pays marqué par une sécheresse permanente. Elle est mise en scène par un narrateur interne, un griot (mais cela n'est pas dit). On peut la résumer ainsi :

Dans un paysage semi-désertique, coupé de montagnes noires, les habitantes d'un village africain sont obligées d'aller chercher quotidiennement l'eau d'un marigot éloigné. Solène, la plus jolie de toutes, est, quoique mariée, toujours sans enfant. Un jour pourtant, une vieille femme à qui elle avait donné à boire lui annonce qu'elle sera bientôt enceinte mais la met en garde contre la pluie dont elle devra protéger l'enfant. Dès sa naissance, cet enfant révèle des dons de musicien extraordinaires. À écouter Nanema jouer du tamtam ou d'un autre instrument tous les habitants du village se mettent à danser « comme envoutés ». Sa renommée va donc grandissant. Une fois adulte, il demande en mariage la fille $\mathrm{du}$ forgeron auquel il paye le douaire traditionnel (vaches et chèvres). Malgré les craintes de sa mère, il jouera de la musique le jour de ses noces. Tous aux alentours se rendent au mariage. Nanema joue de différents instruments puis de sa «flûte enchanteresse » qui fait non seulement danser les humains mais surgir les animaux qui participent à la danse. Les nuages même l'entendent au loin et se joignent à leur tour à la fête en dansant. La pluie alors se met à tomber, pour la première fois, sur Nanema qui se transforme en fleur, et sur la terre où la végétation se met à pousser, les arbres à porter des fruits tandis que les oiseaux s'abritent sous leurs feuillages. Voilà l'origine de la coutume qui veut que l'on offre des fleurs aux femmes que l'on aime, conclut le narrateur.

L'univers du récit qui est celui d'un village africain traditionnel était a priori inconnu des élèves. Le vocabulaire spécifique à cet environnement a donc été expliqué au début des deux séances. Il s'agissait surtout du nom des instruments de musique utilisés par le héros (tam-tam, kora, balafon etc.). Afin d'obtenir une lecture identique lors des deux séances, certaines formulations du texte jugées trop synthétiques ou trop difficiles ont été d'emblée simplifiées ou conservées mais suivies d'une paraphrase explicative ${ }^{11}$.

texte support étant le même, les résultats de ces deux séances devraient être sensiblement identiques avec un gain en termes de mémorisation et de motivation pour la séance sur album, si l'on veut bien suivre l'avis des enseignants de maternelle (Grossmann, déjà cité). Voyons ce qu'il en est sur les plans langagier et linguistique.

\section{Analyse langagière}

Il s'agira ici de donner une image générale de la séance. Nous traiterons donc de la participation des élèves que nous rapporterons à la structure de la séance et nous conclurons sur les thèmes traités par les élèves.

\subsection{Participation des élèves à la séance}

17 La séance B est un peu plus longue (346 tours de parole) que la séance A (309 tours de parole). La différence sur trois cents tours de parole pourrait être tenue pour accidentelle ou négligeable, mais elle correspond bien à une différence entre le nombre des interventions des élèves d'une séance à l'autre : 157 en A et 178 en B. Ceci se traduit par un différentiel de plus de $17 \%$ dans le nombre de mots prononcés au cours des deux séances (tableau ci-dessous). 


\title{
Nombre de mots prononcés par les élèves
}

\author{
in: $A \geqslant B$
}

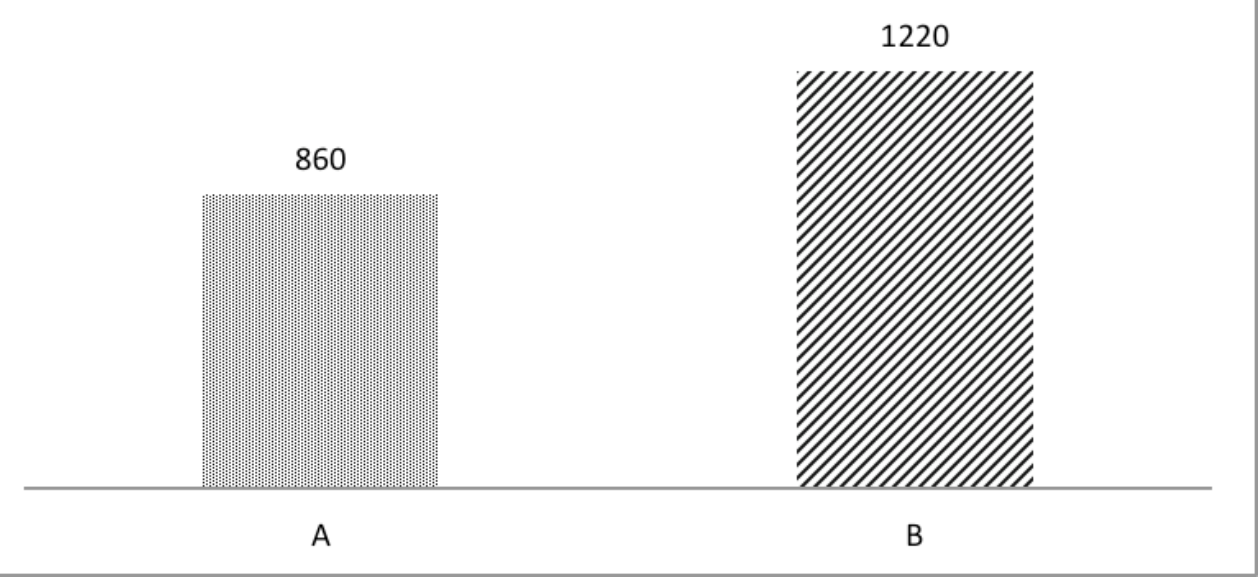

Cette différence ne correspond pas à une simple augmentation du nombre de tours de parole, elle se traduit dans les faits par un allongement des interventions qui bénéficie à la séance $B$ au cours de laquelle les élèves produisent des interventions qui comptent au moins un mot de plus en moyenne : 6,80 en B contre 5,5 en A. Au total, les élèves parlent donc un peu plus souvent et un peu plus longtemps lors de la séance $\mathrm{B}^{12}$.

\subsection{Questionnement et structure des séances}

Les meilleurs résultats de la séance $\mathrm{B}$ ne sont pas rapportables à une conduite différente des échanges au cours des deux séances. Le nombre questions de l'enseignante d'une séance à l'autre est du même ordre : 34 en A et 36 en B; les autres interventions en nombre à peu près équivalent lors des deux séances ont des fonctions de régulation des prises de parole: désignation des locuteurs, incitations à répondre, répétitions d'une réponse d'élève. Enfin, la structure de la séance qui actualise le questionnement présenté plus haut est fondamentalement la même.

Interventions introductrices aux différentes phases de la séance A

\begin{tabular}{|l|l|l|}
\hline $\mathbf{N}^{\circ}$ & Interventions de l'enseignante & $\begin{array}{l}\text { Phases de la } \\
\text { séance }\end{array}$ \\
\hline 1 & $\begin{array}{l}\text { la parole et me raconter ce que vous vous souvenez de l'histoire / } \\
\text { d'accord? }\end{array}$ & Restitution \\
\hline 146 & chht chht/ est-ce qu'on a tout dit sur l'histoire? & Exhaustivité \\
\hline
\end{tabular}




\begin{tabular}{|l|l|l|}
\hline 175 & $\begin{array}{l}\text { alors/ l'histoire que je vous ai lue / Valentin tu écoutes / tu te redresses / } \\
\text { l'histoire que je vous ai racontée/ selon vous/ est-ce que c'est une histoire } \\
\text { vraie ou pas? / est-ce que ça peut arriver dans la réalité ? }\end{array}$ & Réel-Fiction \\
\hline 192 & $\begin{array}{l}\text { voilà / ensuite / maintenant je vais vous poser une autre question / dans } \\
\text { l'histoire / qu'est-ce qui a changé entre le début et la fin de l'histoire ? au } \\
\text { début les gens sont dans une certaine situation et après à la fin }\end{array}$ & Inversion \\
\hline 210 & $\begin{array}{l}\text { le petit garçon / Nanema / s'est transformé en fleur oui / ensuite / une } \\
\text { autre question / qui sont les personnages de cette histoire ? de qui est-ce } \\
\text { qu'on parle dans cette histoire? }\end{array}$ & Personnages \\
\hline 229 & $\begin{array}{l}\text { maintenant on va de nouveau parler sur l'histoire tous ensemble / donc / } \\
\text { donc vous levez le doigt / et je vous interroge / et vous dites ce que vous } \\
\text { vous souvenez de l'histoire / Alexis }\end{array}$ & Restitution II \\
\hline
\end{tabular}

Interventions introductrices aux différentes phases de la séance B

\begin{tabular}{|l|l|l|}
\hline $\mathbf{N}^{\circ}$ & Interventions de l'enseignante & $\begin{array}{l}\text { Phases de la } \\
\text { séance }\end{array}$ \\
\hline 1 & $\begin{array}{l}\text { qu'est-ce qu'il se passe dans l'histoire / de quoi est-ce que vous vous } \\
\text { souvenez? oui Lola? }\end{array}$ & Restitution \\
\hline 170 & $\begin{array}{l}\text { alors / d'abord je vous pose la question hein alors on lève le doigt pour } \\
\text { / l'histoire que je vous ai lue / est-ce que c'est une histoire vraie? }\end{array}$ & Réel-Fiction \\
\hline 194 & $\begin{array}{l}\text { bon / une deuxième question / qu'est-ce qui a changé dans la vie des } \\
\text { habitants entre le début et la fin de l'histoire? au début il se passe / ils } \\
\text { sont dans une certaine situation et après ils : : : }\end{array}$ & Inversion \\
\hline 221 & $\begin{array}{l}\text { dans les arbres / très bien / alors / je vous pose une autre question / qui } \\
\text { sont les personnages de cette histoire? }\end{array}$ & Personnages \\
\hline 258 & $\begin{array}{l}\text { très bien en Afrique / bon maintenant / est-ce que l'on a donné les } \\
\text { réponses à toutes les questions que j'ai posées? }\end{array}$ & $\begin{array}{l}\text { Exhaustivité } \\
\text { sur / alors maintenant j'arrête mes questions et on va de nouveau parler }\end{array}$ \\
\hline Restitution II \\
\hline
\end{tabular}

Le rythme de travail est sensiblement le même, et toutes proportions gardées, les phases ont la même durée avec - on pouvait s'en douter (Colleta, 1994) -, une domination nette des phases consacrées à la restitution du récit (cellules grisées).

\begin{tabular}{|l|l|l|}
\hline Durée des différentes phases (en nombre d'interventions M et Els) & Séance A & Séance B \\
\hline Restitution & 145 & 169 \\
\hline
\end{tabular}




\begin{tabular}{|l|l|l|}
\hline Exhaustivité & 29 & 5 \\
\hline Réel-Fiction & 17 & 24 \\
\hline Inversion & 18 & 27 \\
\hline Personnages & 19 & 37 \\
\hline Restitution II & 81 & 84 \\
\hline Total & 309 & 346 \\
\hline
\end{tabular}

21 La différence au niveau de la phase d'exhaustivité ${ }^{13}$ n'est pas significative, elle correspond en $\mathrm{A}$ à un prolongement de la restitution tandis qu'en $\mathrm{B}$, la restitution était assez productive et la phase d'exhaustivité n'avait plus vraiment de contenu à développer. On peut noter par contre, un meilleur développement des phases intermédiaires RéelFiction, Inversion, Personnages en $\mathrm{B}$, ce qui peut correspondre à une meilleure appréhension de ces questions et donc des thèmes de l'histoire, point que nous allons maintenant aborder.

\subsection{Thèmes repris au cours des deux séances}

Les élèves ont-ils compris cette histoire? Il est difficile de répondre à la question ainsi posée. Peut-on inférer d'une réponse bien formulée mais isolée que d'autres élèves ont compris eux aussi la scène qui vient d'être évoquée ? Il paraît plus sûr de nous demander ce que les élèves se sont rappelés au cours de la séance. Quels sont les thèmes ${ }^{14}$ retenus? Quelles sont les modalités de rappel de ces thèmes?

23 Nous avons identifié 37 thèmes différents repris par les élèves des deux groupes, soit trois à quatre fois moins que ce que l'on obtiendrait en faisant la même chose sur le texte original. C'est peu, mais l'histoire n'a été lue (séance B) ou n'a été lue-présentée (séance A) qu'une seule fois.

On trouve 27 thèmes différents dans les interventions de la séance $\mathrm{A}$ et 32 lors de la séance $B$. Mais les thèmes retenus par les élèves du groupe $B$ sont-ils vraiment plus pertinents pour la construction du récit que ceux, moins nombreux, du groupe A ? Les élèves du groupe $A$ rappellent en effet deux thèmes essentiels omis par ceux du groupe $B$ : le fait que Nanema tombe amoureux (ce qui déclenchera le mariage et la suite des événements climatiques) et d'autre part le fait que la première pluie est à l'origine d'une eau abondante. Mais d'un autre côté, les élèves du groupe B rappellent eux aussi deux thèmes essentiels omis par les autres: l'annonce à Solène de sa future maternité et la mise en garde contre la pluie dont elle devra protéger son enfant. Notre comparaison est d'autant moins discriminante que deux de ces thèmes, un dans chaque séance, n'ont été évoqués que par un seul élève. Aussi pour contourner l'obstacle de l'unicité de certaines réponses, nous n'avons pris en compte que les interventions portant sur des thèmes repris par au moins deux élèves différents. Là encore, il n'y a pas beaucoup de différences, 20 thèmes en $\mathrm{A}$ et 18 thèmes en $\mathrm{B}$. Le nombre de mentions de ces thèmes différents est d'ailleurs équivalent, 115 fois en A et 117 en B. 
De la même manière, les modalités du rappel sont assez proches pour les thèmes les plus souvent rappelés. Figurent en tête des rappels les thèmes dont les termes ont pu faire l'objet d'une reprise directe du texte de la part des élèves. Ainsi dans la séance $\mathrm{A}$, le thème de la demande de l'eau est presque toujours formulé en utilisant l'expression "gorge sèche » présente dans le texte initial, comme son complémentaire, l'offrande de l'eau, est presque toujours formulé en utilisant le mot "gourde», lui aussi présent dans le texte initial. De même dans la séance $B$, le thème des oiseaux cherchant refuge sous les feuilles donne lieu à de multiples interventions qui reformulent d'une manière plus ou moins heureuse le texte initial « chercher refuge sous les feuilles » qui a intrigué les élèves; celui de la naissance de la végétation « des fruits qui poussent sur les arbres » donne lieu lui aussi à de nombreuses reformulations qui témoignent du travail énonciatif effectué par les élèves au cours de la séance.

\begin{tabular}{|l|l|l|}
\hline 58 & Youssef & euh : : les oiseaux i(l)s étaient en dessous des feuilles \\
\hline 62 & Jérôme & les oiseaux i(ls) se protégeaient en dessous des feuilles \\
\hline 136 & Youssef & les oi(seaux / les oi(seaux / les oiseaux se sont / se sont protégés \\
\hline 312 & Gabriel & les oiseaux se sont protégés de la pluie \\
\hline 314 & Chiara & les oiseaux se sont cachés derrière les feuilles \\
\hline 316 & Jérôme & les oiseaux se / se / se sont protégés des feuilles \\
\hline 144 & Lola & l'oiseau s'est protégé dans sa plume \\
\hline
\end{tabular}

26 Ainsi, et cela n'était pas attendu, le mécanisme le plus productif pour intervenir et apporter sa contribution au rappel des différents épisodes de l'histoire est le même pour tous les élèves, il est de s'appuyer sur des éléments du texte initial. Mais à ce jeu, les élèves du groupe B réussissent un peu mieux que ceux du groupe A pour le nombre de thèmes cités. Cela peut laisser supposer qu'ils réemploient plus facilement le matériel lexical présent dans le texte support de séance. Nous allons voir plus précisément ce qu'il en est, en comparant les interventions des élèves sur le plan lexical et syntaxique.

\section{Analyse linguistique}

L'étude proprement dite des interventions des élèves porte sur leurs dimensions linguistiques (\$ 3.1) (lexique, syntaxe). Au passage, nous nous interrogerons sur les modalités de construction de leurs interventions tant sur le plan individuel que collectif.

\subsection{Lexique nominal}

Les élèves utilisent au total 72 noms et adjectifs différents sur les 112 que compte le texte ${ }^{15}$. Dans leur très grande majorité, ces termes appartiennent au vocabulaire habituel des élèves de l'école maternelle tel que le donnent les listes de fréquence auxquelles se réfère 
le site Eduscol ${ }^{16}$. Quelques mots n'en font pas partie, il s'agit de : Afrique, cocotier, tam-tam, pôle sud, pingouin et truc.

Les mots les plus fréquents (au moins dix occurrences au total sur les deux séances) correspondent aux deux épisodes les plus longuement évoqués par les élèves: la rencontre de Solène et de la vieille femme au marigot et le mariage de Nanema au cours duquel sa musique va provoquer le changement climatique et sa propre transformation en fleur. Il s'agit de : vieille, tambour, feuille(s), tam-tam, histoire, gourde, nuages, pluie, arbres, gorge (sèche), oiseaux, eau, garçon et fleur(s). Leur présence quasi obligatoire dans la restitution de l'histoire fait qu'ils ont été employés en nombre à peu près équivalent lors des deux séances : 110 occurrences en A et 138 en $B$.

Les résultats sont différents pour les mots dont la fréquence est plus faible (entre 8 et 2 occurrences au total sur les deux séances). Ils sont deux fois plus nombreux en B (87 occurrences) qu'en A (40 occurrences). Il s'agit en B de termes qui servent à la désignation des personnages : sorcière, Nanema, mémé, maman, madame femme, fille, papa, dame, enfant, monsieur, ou de termes qui désignent des éléments du deuxième plan (description, localisation): noir(e), grande, coquillages, montagne(s), pierre(s), Afrique, maison, village, mariage (au), fête (à la), ou encore de termes désignant des éléments en relation avec les deux autres catégories : soif, chose, instruments, herbe, bébé, fruits. Et ces termes sont repris par plusieurs élèves puisqu'on en a plusieurs occurrences. On voit ici se dessiner une différence essentielle entre les deux séances : une meilleure identification des actants et l'esquisse d'un deuxième plan suffisent pour augmenter le bagage lexical mobilisé lors de la séance $B$.

Les deux séances se différencient aussi par le recours aux lexèmes qui n'ont été utilisés qu'une seule fois. C'est alors la séance $B$ où l'on trouve le moins d'exemples de ce type mais tous sont en rapport avec l'histoire : collier, désert, éclair (orage), fin (de l'histoire), plume (d'oiseaux), robe, tomates (fruits), tronc (d'arbres), trou (d'eau) ou encore incroyable dans une citation du texte original «Il se passa quelque chose d'incroyable ». Il y en deux fois plus, dix-neuf exactement, lors de la séance A. La plupart (12) sont bien en rapport avec l'histoire: musique, tambourin, amoureux, gros ventre (Solène est enceinte), animaux, rochers, buissons, chèvres et vaches, fermier (le forgeron), grande («l'eau est devenue grande », sic), truc; mais les autres (7) supposent d'avoir les illustrations en tête pour être compris : grise (« la pluie est grise » dit un élève mais le texte ne dit rien de tel), cocotier (en fait un arbre du voyageur représenté sur une illustration mais non désigné ainsi dans le texte qui s'en tient au générique arbres), perroquet (identifié ainsi à partir d'une illustration alors que le texte s'en tient au générique oiseaux), enfin plus surprenants mais convoqués selon le même processus : bateau, neige, pingouins et pôle sud (une illustration représente un grand nombre d'animaux différents, y compris d'autres régions du monde, accourant au mariage de Nanema). Le nombre d'occurrences de ces termes à emploi unique qui sont le fait d'élèves différents est l'indice d'un faible taux de reprises entre élèves lors de la séance $\mathrm{A}$, nous y reviendrons. D'un autre côté, le recours à des termes qui ne sont interprétables que par référence aux illustrations marque tout à la fois la puissance mémorielle des illustrations (les élèves ne les ont pourtant vues qu'une fois) et l'obstacle qu'elles peuvent constituer dans le cadre de la narration collective quand elles ne sont pas clairement interprétables. 


\subsection{Lexique verbal} verbes qui appartiennent tous à la liste de fréquence déjà citée. Les verbes jouer et avoir sont les plus fréquents. Le premier (jouer d'un instrument, de la musique etc.) est employé 27 fois en A et 23 fois en B. Pour le second avoir, cela dépend de la construction retenue : composant du présentatif il $y a$, on le trouve 19 fois en A mais seulement 7 fois en B, on reviendra plus loin sur cette différence ; on le trouve aussi souvent dans la séquence avoir soif ou avoir la gorge sèche 8 fois en $\mathrm{A}$ et 3 fois en $\mathrm{B}^{17}$. Les autres verbes, même être, se répartissent très inégalement. Dix-huit ne se trouvent qu'en séance $\mathrm{B}$ : aimer, casser, creuser, croire, déménager, devoir, entendre, exister, oublier, recevoir, rencontrer, rentrer, se passer, dire, vouloir, venir, boire, protéger. Six ne se trouvent qu'en séance A : courir, vendre, pleuvoir, vivre, prendre, raconter ${ }^{18}$. Cette différence se retrouve dans le nombre des occurrences verbales. Si on laisse de côté les deux verbes les plus fréquents jouer et avoir, on trouve 21 verbes différents en A pour 61 occurrences et 33 verbes différents en B pour 101 occurrences, soit un tiers de mieux pour la séance B.

On sait l'importance du verbe dans la construction de la phrase en français. Ces résultats ont donc un impact direct sur la nature et l'intérêt des constructions syntaxiques mobilisées dans les deux séances. Ce que nous allons voir maintenant.

\subsection{Syntaxe}

Pour comparer les interventions faites lors de ces deux séances, nous ne retiendrons pas les réponses en oui ou non, les répétitions strictes à partir de la troisième intervention identique ${ }^{19}$, les interventions répétées à la demande de l'enseignante quand elle n'a pas entendu la réponse, les interventions qui portent sur la situation de communication et non sur l'histoire et enfin les silences, soit pour l'ensemble : 31 interventions en A et 29 en B. Notre analyse portera donc sur 126 interventions en A et 149 en B. Ces dernières témoignent d'une plus grande richesse syntaxique comme on peut le voir dans le tableau suivant (cellules grisées). Le nombre supérieur d'interventions à 1 verbe (en gras) dans la séance A (110) par rapport à la séance B (98) attire d'autant notre attention.

\begin{tabular}{|l|l|l|l|}
\hline Analyse syntaxique & Séance A & Séance B & Total \\
\hline Interventions se ramenant à un groupe nominal & 10 & 32 & 42 \\
\hline Interventions comportant 1 verbe & $\mathbf{1 1 0}$ & $\mathbf{9 6}$ & 206 \\
\hline Interventions comportant 2 verbes & 5 & 16 & 21 \\
\hline Interventions comportant 3 verbes ou plus & 1 & 5 & 6 \\
\hline Nombre total d'interventions prises en compte dans l'analyse & 126 & 149 & 275 \\
\hline
\end{tabular}

Il s'agit en fait d'interventions dont la structure est assez variée comme le montre le tableau suivant dans lequel trois structures (cellules grisées) mériteront un commentaire. 


\begin{tabular}{|l|l|l|l|}
\hline Formes syntaxiques à 1 verbe (206) & Séance A & Séance B & Total \\
\hline Présentatifs (il y a / avait) & 17 & 9 & 26 \\
\hline Le N qui & 2 & 0 & 2 \\
\hline 1 verbe & 8 & 19 & 27 \\
\hline 1 verbe + 1 ou 2 compléments & 83 & 68 & 151 \\
\hline
\end{tabular}

Nous verrons ensuite les interventions à deux et trois verbes, nettement plus nombreuses dans la séance $\mathrm{B}$, comme le montre le tableau ci-dessous.

\begin{tabular}{|l|l|l|l|}
\hline Formes syntaxiques à plusieurs verbes (27) & Séance A & Séance B & Total \\
\hline Présentatifs + qui (il y a / avait X qui) & 3 & 4 & 7 \\
\hline 2 verbes & 2 & 12 & 14 \\
\hline 3 verbes ou plus & 1 & 5 & 6 \\
\hline
\end{tabular}

\subsubsection{Interventions se ramenant à un groupe nominal}

Il y a trois fois plus de réponses nominales lors de la séance $B$ alors que l'écart devrait être de $10 \%$ s'il était proportionnel à la différence entre les nombres d'interventions au cours des séances. Voyons comment ces interventions se mettent en place au cours des échanges.

\begin{tabular}{|l|l|l|l|}
\hline & Interventions se ramenant à un groupe nominal (42) & A & B \\
\hline $\mathbf{1}$ & Réponses immédiates à une question fermée de l'enseignante & 3 & 5 \\
\hline $\mathbf{2}$ & $\begin{array}{l}\text { Introduction d'éléments nouveaux dans le récit. Souvent en réponse à la question } \\
\text { «de quoi vous rappelez-vous?» }\end{array}$ & 2 & 5 \\
\hline $\mathbf{3}$ & Ajout, reformulation ou correction d'une intervention précédente & 2 & 6 \\
\hline $\mathbf{4}$ & Éléments difficiles à formuler & 1 & 2 \\
\hline $\mathbf{5}$ & Réponses données à la question des personnages & 2 & 14 \\
\hline & Total & 10 & 32 \\
\hline
\end{tabular}




\section{Synthèse}

\begin{tabular}{|c|c|c|c|c|}
\hline $\begin{array}{l}\text { Réponses introduites } \\
\text { par un présentatif }\end{array}$ & Séance A. Exemples & & Séance B. Exemples & \\
\hline $\begin{array}{l}\text { Fait référence à tel ou tel } \\
\text { éléments d'une } \\
\text { illustration }\end{array}$ & $\begin{array}{l}\text { il y avait un cocotier / des pingouins / } \\
\text { de la pluie gris }\end{array}$ & 3 & & 0 \\
\hline $\begin{array}{l}\text { Introduction de référents } \\
\text { nouveaux dans l'échange. }\end{array}$ & $\begin{array}{l}\text { il y avait de l'eau fraîche / une gourde } \\
\text { / un XX(rocher?) noir }\end{array}$ & 3 & & 0 \\
\hline $\begin{array}{l}\text { Introduction ou rappel } \\
\text { des différents actants } \\
\text { présents au mariage }\end{array}$ & $\begin{array}{l}\text { les nuages et la pluie (8) ainsi que } \\
\text { d'autres éléments constitutifs de la } \\
\text { scène: oiseaux, arbres, montagnes } \\
\text { noires (3) }\end{array}$ & 11 & $\begin{array}{l}\text { pluie et nuages (3) } \\
\text { autres thèmes, fleurs, } \\
\text { tomates, fruits (3) }\end{array}$ & 6 \\
\hline $\begin{array}{l}\text { Présentatifs à verbe être } \\
\text { dans des propositions } \\
\text { explicatives }\end{array}$ & & 0 & $\begin{array}{l}\text { parce que c'est une } \\
\text { histoire inventée }\end{array}$ & 3 \\
\hline
\end{tabular}

\section{Synthèse}

Le présentatif est plus fréquent lors de la séance $\mathrm{A}^{20}$, on le trouve pour introduire des thèmes nouveaux ce qui est logique mais aussi de manière spécifique à cette séance pour introduire des thèmes d'origine iconique et pour la reprise fréquente du couple pluie / nuages lors de l'épisode de la noce, ce qui a pour effet de juxtaposer des thèmes parallèles plutôt que de les articuler. Ainsi la répétition de la paire pluie / nuages dans l'épisode du mariage.

\begin{tabular}{|l|l|l|}
\hline 207 & Guillaume & y avait des nuages \\
\hline 218 & Kiara & y avait de la pluie qui tombait \\
\hline
\end{tabular}




\begin{tabular}{|l|l|l|}
\hline 234 & Guillaume & y avait des nuages \\
\hline 260 & Alexis & y avait des nuages \\
\hline 286 & Anaïs & il a plu \\
\hline 288 & Els & il y avait des nuages \\
\hline 292 & Els & y avait des nuages \\
\hline 294 & Léon & y avait de la pluie et puis XXX \\
\hline 300 & Alexis & euh :: : y avait de la pluie gris \\
\hline 302 & Guillaume & y avait des nuages \\
\hline
\end{tabular}

43 Les élèves de la séance $\mathrm{B}$ y ont beaucoup moins recours; ils mobilisent une structure à verbe être dans des réponses explicatives et la structure en il y a pour introduire ou rappeler des éléments de la scène des noces.

\subsubsection{Interventions du type <sujet + verbe>}

La formulation binaire de la structure <sujet + verbe> masque les différences de ses actualisations possibles, selon que le sujet soit un nom un pronom. Sur les huit occurrences en séance $\mathrm{A}$, trois interventions ont un sujet pronominal dont le référent nominal est absent des échanges qui précèdent ${ }^{21}$.

\section{Séance A}

\begin{tabular}{|l|l|l|}
\hline 10 & Alexis & y a : : : elle (Solène) est allée chercher l'eau \\
\hline 18 & Kiara & il (Nanéma) était tombé amoureux \\
\hline 160 & Jade & ils (les habitants) ont dansé \\
\hline
\end{tabular}

Une seule intervention s'appuie sur un référent nominal explicite «la femme» dont l'identité n'est cependant accessible qu'aux élèves susceptibles de la rapporter au prédicat lui-même.

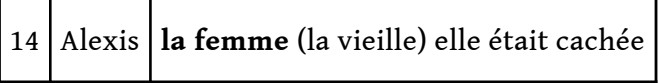

La formulation de cette dernière intervention <thème + sujet + groupe verbal> est celle de la phrase de base à l'oral (Morel \& Danon-Boileau, 1998). Elle apparait plus fréquemment dans la séance $B$, en l'occurrence sept fois vers la fin de la séance ce qui lève le problème de l'identité du référent du groupe nominal déjà donné plusieurs fois (la femme, les oiseaux, l'enfant). 


\section{Séance B}

long de la séance. Elles apparaissent principalement dans trois cas de figures: pour introduire des thèmes nouveaux (3), pour des enchaînements thématiques (6) comme dans la séance A mais aussi pour des reformulations (8) qui peuvent éventuellement poser un problème énonciatif à l'élève et qui alors mériteront un commentaire particulier (2).

Enchaînements thématiques. Lors de la restitution, les élèves reprennent les éléments constitutifs de l'épisode du mariage, qu'ils reformulent les uns après les autres. Le fait d'utiliser des sujets nominaux permet une reconstruction assez correcte de l'épisode, chaque actant identifié faisant l'objet d'une prédication pertinente. Deux cas se présentent, quand le nom n'est pas repris par un pronom, la succession des interventions donne une impression de fluidité, de continuité narrative, comme dans le passage suivant qui rappelle les noces de Nanema.

\begin{tabular}{|l|l|l|}
\hline 122 & Gabriel & euh : : la(...) les oiseaux s'étaient protégés de la feuille \\
\hline 124 & Youssef & les nuages sont à la fête \\
\hline 126 & Emma & les oiseaux ils sont protégés sous les feuilles \\
\hline 128 & Tom & les nuages sont venus au mariage et i(l)s ont dansé \\
\hline
\end{tabular}

Lorsque le nom fait l'objet d'une reprise pronominale, l'impression de continuité narrative disparait pour laisser place à une forme de juxtaposition des informations qui reste cependant compréhensible. Ainsi dans les enchainements 128-134 et 136-138.

\begin{tabular}{|l|l|l|}
\hline 128 & Tom & les nuages sont venus au mariage et i(l)s ont dansé \\
\hline 134 & Gabriel & l'enfant / il s'est marié \\
\hline 136 & Youssef & les oi / les oi / les oiseaux se sont / se sont protégés \\
\hline 138 & Lola & le garçon il a joué de la flûte \\
\hline
\end{tabular}

Reformulations. Lola complète l'épisode de la maternité de Solène amorcé par Tom en 72. En 74, elle évoque d'abord le tam-tam dont Nanema se met à jouer dès sa naissance puis en 76 reformule son intervention à la demande de la maîtresse, ce qui lui vaut un satisfecit (77).

\begin{tabular}{|l|l|l|}
\hline 72 & Tom & $\begin{array}{l}\text { ben / la / la / la maman / y a la / la mémé qui a / qui a dit qu'elle va avoir un bébé } \\
\text { et ben après c'est / elle s'est cachée derrière une maison }\end{array}$ \\
\hline 73 & M & oui elle est partie se cacher dans le village / c'est juste / Lola \\
\hline 74 & Lola & XXX tam-tam \\
\hline
\end{tabular}




\begin{tabular}{|l|l|l|}
\hline 75 & M & pardon ? \\
\hline 76 & Lola & et ben $\mathbf{l}(\mathbf{e})$ petit garçon jouait $:::$ jouait \\
\hline 77 & M & oui très bien / Alessio \\
\hline 78 & Alessio & euh : : : le petit garçon il a joué du tam-tam \\
\hline
\end{tabular}

51 Difficultés. L'absence de complément peut correspondre à une difficulté que le locuteur n'a pas surmontée. Cela se produit au moins deux fois, en 60 et en 199. Ces difficultés témoignent du travail énonciatif mieux que ne peuvent le faire des énoncés plus achevés. En 60, Quentin reprend une information déjà reformulée trois fois de manières différentes juste avant; on peut supposer qu'il tente de faire de même mais qu'il n'y parvient pas.

\begin{tabular}{|l|l|l|}
\hline 54 & Noah & euh / les oi : :seaux sont venus / au maria : :ge \\
\hline 56 & Chiara & les / les / les / oiseaux i(ls) sont allés s(e) protéger sous les feuilles \\
\hline 58 & Youssef & euh / les oiseaux ils étaient en dessous des feuilles \\
\hline 60 & Quentin & euh : : : : les oiseaux / i(ls) sont \\
\hline
\end{tabular}

52 En 197, Gabriel a évoqué la métamorphose du héros devenu fleur au contact de la première pluie. À sa suite, il semble bien qu'un autre élève tente d'évoquer la suite de ce processus par ce qui pourrait être un retour à la situation première « et puis il est de nouveau » mais ce possible narratif n'est pas activé dans le récit et l'énoncé s'arrête là. L'erreur si c'en est une montre bien comment l'élève s'est appuyé sur le propos de son camarade Gabriel pour intervenir à son tour.

\begin{tabular}{|l|l|l|}
\hline 197 & Gabriel & y a le garçon qui est devenu une fleur \\
\hline 198 & M & oui très bien \\
\hline 199 & Els & et puis il est de nouveau \\
\hline
\end{tabular}

\section{Synthèse}

53 Les propositions du type <sujet+verbe> s'inscrivent facilement dans les échanges. Lors de la séance $B$, ces constructions ont très souvent un sujet nominal qui permet plus facilement aux élèves de mettre en place les uns à la suite des autres les différents éléments des épisodes de l'histoire (le mariage, la naissance de la végétation). Du coup, la restitution de ces épisodes donne lieu lors de la séance $\mathrm{B}$ à des séquences thématiques cohérentes plus longues que ce que l'on pouvait trouver lors de la séance A. 


\subsubsection{Interventions du type <sujet + verbe+ 1 ou 2 compléments $>$} dans 21 interventions sur les 83 mobilisant cette structure. Mais leur nombre est sans doute trop faible pour que leur présence éparse suffise à clarifier les chaînes de référence possibles. Les interventions se juxtaposent sans que les relations puissent être établies sur un plan grammatical voire sémantique. Ainsi dans les enchaînements suivants :

\begin{tabular}{|l|l|l|}
\hline 43 & Kiara & elle a joué de la flûte \\
\hline 45 & Anaïs & elle avait(...) elle avait la gorge toute sèche \\
\hline 47 & Alexis & y avait un XX noir \\
\hline 49 & Timéo & il a joué du tambour \\
\hline 52 & Els & tam-tam ! \\
\hline 55 & Els & l'eau elle est devenue plus grande \\
\hline 57 & Jeanne & il a vendu des chèvres aussi \\
\hline
\end{tabular}


Séance B. des pronoms ayant un référent explicite (structure <thème + pronom + GV>) soit des noms déterminés par un article défini. Ces interventions facilitent la restitution des épisodes de l'histoire (ici celui du mariage) en construisant des référents sémantiquement identifiables. L'enchaînement des interventions-élèves de 82 à 96 (sauf la 86 qui renvoie à un autre épisode) illustre bien cela.

\begin{tabular}{|l|l|l|}
\hline 82 & Emma & le petit garçon il a joué de la flûte \\
\hline 84 & Tom & les oiseaux se sont protégés sous les feuilles \\
\hline 86 & Emma & en fait la mémé eh bien elle : : : en fait la mémé elle demandait de l'eau \\
\hline 88 & Youssef & $\begin{array}{l}\text { i(l) / i(l) / il jouait du tam-tam et / et / et / et tous les gens du village ils ve / ils } \\
\text { venaient danser }\end{array}$ \\
\hline 90 & Noémie & euh / les arbres ils ont euh : : des fruits \\
\hline 92 & Quentin & euh / il a joué de la flû : :te \\
\hline 94 & Gabriel & le petit garçon il XX \\
\hline 96 & Clara & en fait les duages / les nuages ils ont dansé \\
\hline
\end{tabular}

\subsubsection{Interventions à plusieurs verbes}

Dans la séance A, il y a 6 interventions à plusieurs verbes, leur longueur moyenne est de 10 mots. Dans la séance B, il y a 21 interventions de ce type, leur longueur moyenne est de 30 mots. La capacité des élèves à reprendre ou non ce qui qui a déjà été dit est la clé de cette disparité. Sur les 21 interventions à plusieurs verbes que compte la séance B par exemple, 19 sont le fruit du développement de propositions déjà faites par d'autres élèves ${ }^{22}$.

\section{Séance B}

Le thème peut être repris à partir d'une question de l'enseignante (3 fois). Ainsi dans l'exemple suivant, Tom explique qu'on ne connaît pas le nom du père du héros parce qu'on ne l'a jamais entendu parler signant ainsi de manière involontaire mais claire la nature verbale de sa mémorisation.

\begin{tabular}{|l|l|l|}
\hline 244 & Tom & le papa \\
\hline 245 & M & oui le papa aussi \\
\hline 249 & Youssef & la / la maman \\
\hline
\end{tabular}




\begin{tabular}{|l|l|l|}
\hline 250 & M & la maman / est-ce que vous vous souvenez de leur prénom ? \\
\hline 251 & Els & non : : : \\
\hline 252 & Tom & $\begin{array}{l}\text { on sait pas du papa / là on sait pas du papa parce que le papa on l'a pas } \\
\text { entendu parler }\end{array}$ \\
\hline
\end{tabular}

En général, les élèves s'appuient sur ce qui a déjà été dit par leurs camarades. Le plus simple est de reformuler une intervention. C'est le cas ci-dessous pour Gabriel (197)

\begin{tabular}{|l|l|l|}
\hline 42 & Tom & il devient / il / celui-là qui s'est marié / il devient une fleur \\
\hline 102 & Lola & et le petit garçon ça fait une / des fleurs \\
\hline 197 & Gabriel & y a le garçon qui est devenu une fleur \\
\hline
\end{tabular}

61 Dans les interventions les plus longues de la séance $B$, les élèves ne s'en tiennent pas là et introduisent un thème nouveau ou une séquence nouvelle à la suite de la reformulation d'une autre intervention. En voici plusieurs exemples (la séquence nouvelle est en gras tandis que la reformulation est soulignée)

\begin{tabular}{|l|l|l|}
\hline 73 & $M$ & oui elle est partie se cacher dans le village / c'est juste / Lola \\
\hline 76 & Lola & et ben l(e) petit garçon jouait : : : : jouait \\
\hline 88 & Youssef & $\begin{array}{l}\mathrm{i}(\mathrm{l}) / \mathrm{i}(\mathrm{l}) / \mathrm{il} \text { jouait du tam-tam } \text { et / et / et / et tous les gens du village ils ve / ils } \\
\text { venaient danser. }\end{array}$ \\
\hline
\end{tabular}

Dans les deux exemples suivants on voit comment procède Youssef (154) et Alessio (302) pour reformuler ce qui a déjà été dit et le développer par l'ajout d'une nouvelle information.'

\begin{tabular}{|l|l|l|}
\hline 148 & Tom & eh ben : : la sorcière elle voulait boire de l'eau \\
\hline 154 & Youssef & $\begin{array}{l}\text { la sor / sorcière elle voulait boire de l'eau parce qu'elle avait la gorge sèche } \\
\text { comme de la pierre }\end{array}$ \\
\hline 292 & Gabriel & la vieille elle voulait boire de l'eau \\
\hline 302 & Alessio & $\begin{array}{l}\text { euh / la sorcière il / il / il / il / elle / elle avait la gorge toute / il / il / elle avait la } \\
\text { gorge / elle avait la gorge en pierre et la gorge sec(he) et / et / et elle avait soif }\end{array}$ \\
\hline
\end{tabular}

63 C'est dire que non seulement les reprises ou les reformulations sont le support $d u$ développement des interventions ce qui est en soi positif mais c'est aussi l'indice d'un contrôle de la conduite discursive mobilisée qui se traduit lors de l'ajout terminal d'une information nouvelle. Cet apprentissage des conduites discursives est particulièrement 
repérable dans l'intervention d'un élève comme Tom, le meilleur locuteur du groupe qui est un des rares capables de reformuler une partie de ses propres interventions pour les développer, éventuellement en s'appuyant aussi sur ce qu'on dit ses camarades pour obtenir un développement synthétique qui relève clairement du discours monologal.

\begin{tabular}{|l|l|l|}
\hline 72 & Tom & $\begin{array}{l}\text { ben : : la / la / la maman / y a la / la mémé qui a / qui a dit qu'elle va avoir un bébé } \\
\text { et ben après c'est / elle s'est cachée derrière une maison }\end{array}$ \\
\hline 201 & Tom & $\begin{array}{l}\text { au début de l'histoire eh ben / eh ben / eh ben il a eh ben / eh ben / l'enfant il joue } \\
\text { du tam-tam et après / et après / le petit /et après quand la fille il a eu / il a eu une } \\
\text { sorcière elle disait/ elle disait qu'elle voulait boire et après et après elle disait tu vas } \\
\text { avoir un bébé dans neuf mois et y a et après elle est allée / elle est allée quelque part } \\
\text { dans le village }\end{array}$ \\
\hline
\end{tabular}

\section{Séance A}

Les interventions à plusieurs verbes dans la séance A sont moins nombreuses. Leur construction confirme l'hypothèse selon laquelle les élèves ne construisent pas ensemble leur propos, même s'ils s'écoutent, la présence d'une causale ou encore d'anaphoriques pronominaux en étant des indices assez sûrs. Mais l'emploi du présent ( 2 fois) et la présence dans cette structure de présentatifs ( 4 fois) laissent penser que le repère mémoriel de ces énoncés n'est pas tant de nature verbale qu'iconique.

\begin{tabular}{|l|l|l|}
\hline 4 & Alexis & il y a des animaux qui courent sur le bateau \\
\hline 152 & Maëlle & c'était / c'était lui qui avait raconté l'histoire \\
\hline 218 & Kiara & y avait de la pluie qui tombait \\
\hline 187 & Kiara & ils vont cueillir / ils / ils vont donner des fleurs \\
\hline 179 & Léon & parce que à la fin i(l) y a le garçon qui se transforme en fleur \\
\hline 195 & Alexis & ils ont des arbres / ils ont de l'herbe et / et ils ont des oiseaux \\
\hline
\end{tabular}

\section{Discussion}

Les élèves à qui l'on a proposé la lecture-présentation d'album réussissent moins bien que leurs camarades à qui l'on a simplement lu le texte de cette histoire recopié sur une feuille de papier. Leur participation à la séance est inférieure à celle de leurs camarades du groupe B qui parlent plus souvent et plus longtemps qu'eux. Les résultats sur le plan lexical et syntaxique sont tout aussi nets. Le seul domaine où ils font jeu égal, mais pas plus, est celui du nombre et de la variété des thèmes introduits au cours de la séance. Cette première conclusion repose sur les données quantitatives de l'étude mais il en est une autre peut-être plus intéressante. L'analyse linguistique des interventions montre que les élèves des deux groupes ne construisent pas leurs interventions individuellement 
et collectivement de la même manière. Il est apparu au fil de l'analyse que les élèves du groupe A construisent des énoncés plus brefs, très souvent sur la base de présentatifs, avec des sujets pronominaux dont la référence est instable. Pour ces différentes raisons, l'introduction de leurs interventions se fait sur le mode de l'accumulation ou plutôt de la juxtaposition sans développement collectif. Or ces caractéristiques sont clairement celles d'un langage qui se construit sur la base de référents perceptibles et partagés, autrement dit du langage en situation ${ }^{23}$. Les images vues ont cette puissance mémorielle qu'on leur attribue à juste titre et tous les élèves de grande section n'ont pas les capacités de s'abstraire de cette connaissance perceptive partagée et n'ont donc pas les moyens de produire l'énonciation décrochée ${ }^{24}$ qu'en réalité on attend d'eux dans cette situation. A l'inverse, on voit comment les élèves du groupe $B$ témoignent à de nombreuses reprises et à différents niveaux de leur travail de l'origine verbale de leur restitution de l'histoire. Le matériel lexical plus varié et en plus grand nombre autorise des reprises plus faciles entre élèves. On peut faire l'hypothèse que la proximité entre le texte source et les interventions entendues favorise ces reprises et l'allongement des interventions. Contrairement à l'album, la lecture du texte non illustré détermine une meilleure clarté cognitive relativement à ce qui est attendu et aux moyens d'y parvenir, en l'occurrence la centration sur le discours, celui du narrateur d'abord, celui des autres élèves ensuite.

66 A la lumière de ces résultats, il apparaît que la question qui mérite d'être posée n'est plus «Quel album?» pour laquelle les réponses ne manquent pas mais plus largement «Quel support : contage, texte, album?» pour laquelle, hormis l'article de Boiron (2014) déjà cité, les recherches sont assez rares.

\section{BIBLIOGRAPHIE}

Boiron, V. et Rebière, M. (2009). « Quels albums pour la petite section ? Propositions de critères de choix ». Diptyque, $\mathrm{n}^{\circ}$ 17, p. 11-26.

Boiron, V. (2014). « Raconter et lire des récits de fiction : effets comparés sur la compréhension d'élèves de maternelle », Repères, $n^{\circ}$ 50, p. 83-104

Brigaudiot, M. (1993). «Quelques remarques à propos du récit et des images à l'école maternelle ». Repères, $\mathrm{n}^{\circ}$ 7, p. 7-22.

Canut, E., Brunesseaux-Gauthier, F. et Vertalier, M. (2012). Des albums pour apprendre à parler. Les choisir, les utiliser en maternelle. Nancy : Scéren / CRDP.

Colleta, J-M. (2004). Le développement de la parole chez l'enfant âgé de 6 à 11 ans Corps, langage et cognition. Wavre (Belgique) : Mardaga.

Ducancel, G. (dir.). (2006). Sens et code au cycle 2 Apprentissages progressifs de l'écrit au cycle 2, Paris : INRP / Hachette éducation.

Francois, F., Francois, D., Sabeau-Jouannet, E. et Sourdot, M. (1977). La syntaxe de l'enfant avant 5 ans. Paris : Larousse

Grossmann, F. (1996). Enfances de la lecture. Berne-Paris : Peter Lang. 
Hickmann, M. (2002) « Développement de la production verbale orale ». In M. Fayol [dir.], Production du langage. Paris : Hermès Science, p. 173-190.

Lentin, L. (1983). L'album pour enfants non-lecteurs. Paris : Presses de la Sorbonne nouvelle.

Morel, A-M \& Danon-Boileau, L., (1998), Grammaire de l'intonation. L'exemple du français oral, Paris, Ophrys.

Péroz, P., (2014), « parce que son papa, madame Grégory a fait un civet, un civet avec le papa » Sur les procédures d'apprentissage du lexique : reprise et reformulation en langage oral à l'école maternelle. Congrès Mondial de Linguistique Française, 19-23 juillet 2014, Berlin, disponible sur http://www.shs-conferences.org/articles/shsconf/pdf/2014/05/shsconf_cmlf14_01221.pdf Sole, M-R. \& Soler, O. (2010). «L'effet de l'étayage sur les récits des enfants ». Dans J. Bernicot, E.Venezanio, M. Musiol et A. Bert-Erboul (dir.). Interactions verbales et acquisition du langage. Paris : L'Harmattan, p. 45-64.

Stoeckle, R. (1999). L'album à l'école et au collège. Paris : L'Ecole.

Tauveron, C. (2002). Lire la littérature de jeunesse à l'école. Paris : Hatier.

Van Der Linden, S. (2006), Lire l'album, l'atelier du poisson soluble.

Veneziano, E. (2009). «Peut-on aider l'enfant à mieux raconter? Les effets de deux méthodes d'intervention : conversation sur les causes et modèle de récit ». Dans H. Makdissi, A. Boisclair et P. Sirois (dir.). La littératie au préscolaire : une fenêtre ouverte sur la scolarisation. Québec : Presses de l'Université du Québec, p. 107-144.

\section{NOTES}

1. C'est le cas de la majorité des enseignantes qui ont répondu aux questionnaires de Grossmann (1996 : 183). Pour elles, le travail à partir d'un livre a pour principal objectif de «susciter une activité de langage ». C'est aussi le point de vue de la majorité des enseignants de maternelle que nous avons pu recevoir en formation continue.

2. Lily Boulay est l'auteure de recueils de contes comme Magie du conte puis Miroir des contes publiés chez A. Colin et qui étaient présents dans la plupart des écoles maternelles dans les années 80 .

3. La récente liste d'ouvrages recommandés par le Ministère de l'éducation nationale pour le cycle 1 confirme cette évolution : elle compte plus de 230 références d'albums pour seulement 4 recueils de contes.

4. MEN, 2006, Le langage à l'école maternelle, document d'accompagnement des programmes, Scéren/CNDP.

5. L'âge moyen des élèves est de cinq ans en grande section de maternelle. L'année suivante, ils entrent à l'école élémentaire, au cours préparatoire où commence l'apprentissage proprement dit de la lecture.

6. Une première version de cette étude a fait l'objet d'une relecture critique de M. Laparra (MCF, Université de Lorraine), qu'elle en soit ici remerciée.

7. Ecole maternelle de Diebling (57980).

8. D. Kipper, à l'origine de la transcription des deux séances dans son mémoire de master, à l'IUFM de Lorraine, en 2012, Pédagogies du langage oral à l'école maternelle. Comparaison de deux classes de GS.

9. Les deux groupes ont été équilibrés autant que possible. Cependant, l'enseignante titulaire pensait a priori que le groupe A pourrait être un peu plus performant puisqu'il comportait plus 
d'élèves habituellement très bons parleurs que le groupe $B$ qui comportait trois élèves plus jeunes que ceux du groupe A.

10. L'origine de cette histoire est un conte ivoirien rapporté par Innocent YAPI, conteur professionnel installé en Alsace.

11. Notre objet d'étude se limite aux données langagières et linguistiques dans les interventions des élèves, nous n'aborderons donc pas la question des apprentissages culturels que l'on peut identifier ici ou là. Nous ne cherchons pas non plus à évaluer directement la " compréhension » de l'histoire en fonction des modalités de présentation du récit. Nous n'avions pas les moyens de mettre en œuvre le dispositif nécessaire qui est beaucoup plus lourd, comme on pourra le constater en se reportant à l'article de V. Boiron (2014) déjà cité. Nous nous en sommes tenus à obtenir une aussi grande similitude que possible des groupes et des procédures mises en œuvre pour ensuite analyser les éléments auxquels nous avions directement accès : les interventions des élèves dans leur succession au cours des séances de langage qui suivaient la présentation de l'histoire.

12. Les élèves de la classe ne sont pas habitués à écouter des textes lus non illustrés, pourtant ils ont participé régulièrement et activement à la séance $\mathrm{B}$, comme le montrent ces résultats qui remettent en cause l'opinion assez répandue chez les enseignants selon laquelle « il est difficile aux enfants de maternelle de maintenir leur attention à la seule écoute du texte lu » (Tauveron, $2002: 133)$.

13. La phase « exhaustivité » correspond pour les élèves à la recherche de «tout » ce qui n'a pas été encore rappelé à propos de l'histoire. La consigne peut apparaître à différents moments de la séance.

14. Nous nommons «thèmes ", l'ensemble des informations relatives aux personnages, aux lieux et aux événements qui constituent le récit qui, après découpage, se trouve constitué d'une centaine de "thèmes" différents. A titre d'exemple, parmi les thèmes repris ont trouve : l'histoire se déroule en Afrique / le pays est entouré de montagnes noires / Solène est la plus jolie femme du village / elle doit aller chercher l'eau au puits / elle y rencontre une vieille femme / etc.

15. On n'a pas tenu compte ici des noms propres, comme Etangi (tribu des) ou les toponymes comme Mokobé (les monts du) ou Morongo (plateau du). Par contre, on a compté les noms des personnages principaux Solène (non repris) et Nanéma (repris) de même que les noms des instruments de musique comme balafon ou sunza qui avaient été présentés en début de séance. De fait, les enfants ne réutiliseront que tam-tam et flûte.

16. Le vocabulaire et son enseignement. Comment enseigner le vocabulaire en maternelle. (Ressources pour l'école primaire) sur eduscol.education.fr/vocabulaire

17. Dans les autres cas, moins nombreux, le verbe avoir a le sens de porter ou de posséder. Ces emplois sont relatifs à la maternité (avoir un bébé, un gros ventre, un enfant) pour 4 occurrences ou à la possession (avoir une gourde, des arbres, des oiseaux) pour 5 occurrences.

18. Tandis que treize autres se retrouvent dans les deux séances: demander, cueillir, pouvoir, tomber, se transformer, cacher (se), chercher, devenir, marier (se), pousser, faire (se), danser, aller avec cependant un nombre d'emplois supérieur en B pour huit d'entre eux.

19. Dans la série suivante : 85 / Jade / elle avait la gorge toute sèche. 91 / Valentin / elle avait la gorge toute sèche. 93 / Naomie / la vieille/ elle avait la gorge toute sèche. 97 / Guillaume / elle avait la gorge toute sèche. L'intervention 97 est la troisième identique à l'intervention 85 (après la 91); elle n'est donc pas prise en compte dans les décomptes de cette partie d'analyse syntaxique.

20. La fréquence du présentatif lors de la séance A est une des caractéristique d'un discours en situation. Ce point qui est à rapprocher d'autres observations de nature linguistique (lexique et syntaxe) est développé dans la discussion. 
21. Les autres ont pour sujet un pronom impersonnel: «il a plu», "il pleuvait» ou un démonstratif « ça peut arriver ».

22. Cette procédure n'est pas le signe d'une fragilité énonciative, au contraire, elle est le moyen auquel les enfants de 4 et 5 ans ont majoritairement recours pour introduire des informations nouvelles dans des interventions structurées. (Péroz, 2014).

23. Le type de « récits produits à partir d'histoires en images : et pour lesquels on constate que jusque vers 6 ans, le pronom personnel sujet et le déterminant défini sont essentiellement utilisé en production pour désigner un élément présent dans le contexte situationnel (emploi exophorique ou déictique) et sont traités comme tels en compréhension » (J. Karmiloff-Smith, 1981, citée par J-M. Colletta, 128).

24. On sait que cela suppose une maîtrise du « déplacement de la référence » qui n'est acquise par la majorité des élèves qu'à partir de 6 ans d'après M. Hickmann (2002).

\section{RÉSUMÉS}

Les enseignants privilégient l'album comme support pour les séances de langage à l'école maternelle. Il est communément admis que les illustrations facilitent la mémorisation et la compréhension des histoires lues aux élèves et que partant de là elles soutiennent l'apprentissage du langage oral. S'agit-il pour autant du meilleur support pour le développement des compétences langagières et linguistiques des élèves? L'auteur compare deux séances de langage organisées dans des conditions aussi proches que possible dans une classe de grande section, l'une sur album et l'autre à partir du texte extrait de l'album et recopié sur une feuille. Les résultats obtenus diffèrent selon le support de séance. Les enfants qui ont travaillé à partir de l'album ne se souviennent pas mieux de l'histoire et leurs productions langagières sont inférieures à celles du groupe ayant simplement écouté l'histoire. L'analyse des enchaînements des interventions à partir de critères linguistiques (syntaxe et lexique) montre que les modalités mêmes de construction des interventions diffèrent. L'auteur fait l'hypothèse que ces différences peuvent être mises en relation avec la présentation différente de l'histoire: partiellement iconique d'un côté, strictement verbale d'un autre.

The teachers favor the album as the medium for the sessions of language to the nursery school. It is collectively admitted that the illustrations facilitate the memorization and the understanding of the stories read to the pupils and that leaving from there they support the learning (apprenticeship) of the oral language. Is it for all that about the best medium for the development of the linguistic and linguistic skills of the pupils? The author compares two sessions of language organized in conditions so close as possible in a class of big section, the one on album and the other one from the text extracted from the album and copied out on a sheet. The obtained results differ according to the medium of session. The children who worked from the album do not remember better the history and their linguistic productions are lower than those of the group having simply listened to the story. The analysis of the sentences of the interventions from linguistic criteria (syntax and lexicon) shows that the modalities of construction of the interventions differ. The author makes the hypothesis that these differences can be got in touch with the display different from the story: partially iconic on one side, strictly verbal of an other one. 
INDEX

Mots-clés : éducation pré-élémentaire ; apprentissage de la langue maternelle ; didactique ; apprentissage de groupe ; processus d'apprentissage.

Keywords : pre-elementary education; mother tongue learning; didactics; group learning, learning process;

\section{AUTEUR}

\section{PIERRE PÉROZ}

pierre.peroz@univ-lorraine.fr

Chercheur associé

Centre de Recherche sur les Médiations

Université de Lorraine 